\title{
Nuclear-cytoplasmic PARP-1 expression as an unfavorable prognostic marker in lymph node-negative early breast cancer: 15-year follow-up
}

\author{
PIOTR DONIZY ${ }^{1}$, GRAZYNA PIETRZYK ${ }^{4}$, AGNIESZKA HALON ${ }^{1}$, CYPRIAN KOZYRA $^{5}$, \\ TSERENCHUNT GANSUKH ${ }^{6}$, HERMANN LAGE $^{7}$, PAWEL SUROWIAK ${ }^{2}$ and RAFAL MATKOWSKI ${ }^{3,8}$ \\ Departments of ${ }^{1}$ Pathomorphology and Oncological Cytology, ${ }^{2}$ Histology and Embryology, and ${ }^{3}$ Oncology and \\ Division of Surgical Oncology, Wroclaw Medical University, 50-556 Wroclaw; ${ }^{4}$ Laboratory of Mammotomic Biopsy, \\ 4th Military Hospital, 50-001 Wroclaw; ${ }^{5}$ Department of Statistics, Wroclaw University of Economics, 53-345 Wroclaw, \\ Poland; ${ }^{6}$ Medical Research Institute of Mongolia, 210526 Ulan Bator, Mongolia; ${ }^{7}$ Institute of Pathology, Charité \\ Universitätsmedizin, D-10117 Berlin, Germany; ${ }^{8}$ Lower Silesian Oncology Centre, 53-413 Wroclaw, Poland
}

Received December 1, 2013; Accepted January 24, 2014

DOI: $10.3892 /$ or.2014.3024

\begin{abstract}
PARP-1 plays an important role in DNA damage repair and maintaining genome integrity by repairing DNA single-strand breaks (SSBs) by base excision repair (BER). The aim of the present study was to examine the expression of PARP-1 in breast cancer (BC) patients and to assess the relationship between the subcellular localization of this protein and clinicopathological characteristics. The reactivity of PARP-1 was analyzed by immunohistochemistry in a homogeneous group of 83 stage II ductal BC patients with a 15-year follow-up. Immunostaining of PARP-1 was also evaluated in 4 human $\mathrm{BC}$ cell lines and resistance prediction profile for 11 anticancer agents was performed using 3 models of drug-resistant cell lines. Nuclear-cytoplasmic expression (NCE) was associated with shorter overall survival, which was not statistically significant during the 10-year follow-up but became statistically significant after 10 years of observation, during the 15-year follow-up $(\mathrm{P}=0.015)$. Analysis performed in subgroups of patients with $(\mathrm{N}+)$ and without $(\mathrm{N}-)$ nodal metastases showed that NCE was associated with poor clinical outcome in $\mathrm{N}$ - patients $(\mathrm{P}=0.017)$. Multivariate analysis confirmed a significant impact of NCE on unfavorable prognosis in N-early BC. The presence of PARP-1 NCE may be a new potential unfavorable prognostic factor in lymph node- negative early BC.
\end{abstract}

Correspondence to: Dr Piotr Donizy, Department of Pathomorphology and Oncological Cytology, Wroclaw Medical University, Borowska 213, 50-556 Wroclaw, Poland

E-mail: piotrdonizy@wp.pl

Key words: PARP-1, long-term prognosis, breast cancer, immunohistochemistry

\section{Introduction}

Studies using molecular biology techniques confirmed that breast cancer (BC) is a complex disease whose heterogeneity and clinical-therapeutic implications must be determined by new prognostic and predictive markers to personalize therapy to individual patients. Identification of BC subgroups with a different clinical course and different response to systemic treatment is crucial for the improvement of therapy results and will help introduce new, molecularly targeted treatment options to the standard regimen. In line with the St. Gallen expert consensus (1), the evaluation of ER, PgR, HER-2 status and proliferation index measured by $\mathrm{Ki}-67$ immunoreactivity is key as regards prognostic value, in addition to TNM classification.

PARP-1 was identified by Chambon et al (2) in 1963 as a protein whose enzymatic activity allows it to generate ADP-ribose polymers. Gene encoding PARP-1, one of the housekeeping genes, sits on the long arm of chromosome 1 and spans 23 exons (3). PARP-1 protein comprises three main domains: i) DNA binding domain (DBD), located at the N-terminal end of the protein; ii) automodification domain (AMD) and iii) catalytic domain (CD), which spans the C-terminus of PARP-1 molecule (4-7). DBD is composed of three zinc finger motifs (FI-III/Zn1-3) that direct PARP-1 binding to DNA (FI/Zn1 and FII/Zn2) and are involved in interdomain and protein-protein interactions, which is crucial for DNA-dependent enzyme activation (FIII/Zn3) $(8,9)$. AMD is important for the proper biological functioning, and encompasses approximately 15 fragments (mainly glutamic acid) which are sites of PAR chains attachment. CD is situated at the C-terminus of PARP-1 molecule and is responsible for $\mathrm{NAD}^{+}$binding and subsequent elongation and branching of PAR $(3,10)$.

Studies conducted to date suggest an important role of PARP-1 in repair of single-strand breaks (SSBs) by base excision repair (BER). The role of PARP-1 in other repair 
mechanisms requires further analysis due to contradictory reports $(7,11)$. The so-called damage sensor model is proposed in which activated PARP-1 identifies DNA breaks (by DBD) and temporarily binds to the ends of the damaged DNA. With the use of electron microscopy, studies showed that PARP-1 in the form of homodimer interacts electrostatically with DNA covering 7 nucleotides up and down the damage site and is a specific sensor that leads the recruitment machinery to the site of damage $(6,12)$. PARP-1's enzymatic activity can increase 500 -fold on its binding to the damaged DNA, which results in quick elongation of PAR chain and additional automodification of PARP-1 with ADP-ribose, inhibiting enzymatic activity (negative feedback) and detachment of PARP-1 from DNA chain, which ensures further repair of the damage by certain proteins (13).

It was also shown that ADP-ribosylation of histones (mainly $\mathrm{H} 1$ and $\mathrm{H} 2 \mathrm{~B}$ ) ensures loosening of densely packed chromatin structure and recruitment of repair machinery, which is necessary for the adequate correction of DNA changes $(14,15)$. It is also worth noting that most enzymatic activity $(\sim 85 \%)$ is attributed to PARP-1; however, this polymerase is supported in its function of damage signaller by PARP-2, which is necessary to ensure effective BER (16).

Inhibition of PARP-1 enzymatic activity by specific inhibitors results in genome instability, and promotes the occurrence of various DNA disorders, which eventually results in cell death. The other area of research into PARP inhibitors is related to their synergistic activity with genotoxic cytostatic agents and radiotherapy $(16,17)$.

To date, no predictive factor has been identified that would be a reliable qualifier for potential inclusion of PARP inhibitor therapy in BC treatment. Immunohistochemical assessment of PARP-1 reactivity in BC cells and the subsequent decision on starting the therapy or exclusion of the patient may be an alternative, as in the case of HER-2. The above hypothesis needs to be confirmed in extensive, multicentre research studies.

The significance of PARP-1 immunoreactivity in breast carcinogenesis and the impact on long-term survival remains thus far unclear. In the present study, we investigated the prognostic value of PARP-1 protein expression by immunohistochemical analyses in 83 patients with stage II ductal BC. We assessed the relationship between the subcellular localization of these proteins in conjunction with the pathological and clinical characteristics of the patients studied. Considering the favorable prognosis of patients diagnosed with BC (5-year survival rate of almost $90 \%$ ) and recurrence that is often observed after as many as 20 years after the original diagnosis and treatment, we sought to study a group of patients observed for a period of over 5 years. A unique, clinically and therapeutically homogeneous group was enrolled in the study, with accurately documented 15 -year observations.

Patient clinical history was analyzed for a period of 15 years which is an exceptionally long observation period and adds considerable value to the research, but that, however, gives rise to problems relating to the evaluation of predictive value of PARP-1. Fifteen years ago different therapeutic methods were used, namely, more radical surgeries, different radiotherapy schedules and hormonal therapy was applied without the knowledge of steroid receptor expression. The above makes it impossible to apply predictive test results to the currently treated patients; however, the analysis of such a long clinical course provides important information on the prognostic role of PARP-1 expression in BC. It must be stressed that prognostic value of PARP-1 expression in such a homogeneous group or in the group with such long clinical observations has not previously been described.

\section{Materials and methods}

Patients. Tissue samples were obtained from 83 patients treated radically for stage II ductal $\mathrm{BC}$, diagnosed between 1993 and 1994 in the Lower Silesian Oncology Centre in Wroclaw, Poland. The mean age of the patients was 55.2 years. The patients were selected based on the availability of tissues. All patients underwent surgery (Madden mastectomy) with or without adjuvant treatment. Following the applied treatment, the patients were subjected to continuous monitoring in the Lower Silesia Oncology Centre. Data related to relapse and mortality were accumulated using medical documentation available in the Lower Silesia Oncology Centre. Overall survival (OS), cancer-specific overall survival (CSOS) and disease-free survival (DFS) rates were established for all patients. The total number of patients included was stipulated by a single series performed by our institution, the follow-up period of 15 years, and the highly homogeneous characteristics of the tumors selected (ductal invasive BC, G2 and G3, clinical stage II according to UICC, Madden mastectomy). Detailed characteristics of the patient cohort are listed in Table I. This study was approved by the Institutional Review Board of the Wroclaw Medical University, Poland.

Tumor samples. Tumor specimens were fixed in $10 \%$ buffered formalin and embedded in paraffin. All hematoxylin and eosin (H\&E) stained sections were examined by two pathologists. Due to the absence of a population-based BC screening at the time the present study was initiated, the size of the primary tumors was different from the detriment of the value of other, including European countries. The median tumor size was also determined by the inclusion of a homogeneous group of clinical stage II BC (see above). Tumor stages were assessed according to the TNM classification system (18). The tumor grades were estimated according to the Bloom-Richardson protocol, with the Elston and Ellis (19) modification (Table I).

Immunohistochemistry. Immunohistochemical analyses were performed retrospectively on tissue samples collected for routine diagnostic purposes. Formalin-fixed, paraffinembedded tissue sections were freshly prepared $(4 \mu \mathrm{m})$. Immunohistochemistry was performed as previously described (20-22). For the detection of PARP-1, a polyclonal rabbit antibody (clone ab6079; Abcam, Cambridge, UK) was diluted 1:150 in the Antibody Diluent, Background Reducing (DakoCytomation, Warsaw, Poland). For the detection of the estrogen receptor, an optimally pre-diluted monoclonal mouse antibody was used (clone 1D5; DakoCytomation, Glostrup, Denmark) and for the detection of the progesterone receptor, an optimally pre-diluted monoclonal antibody (clone PgR636, DakoCytomation) was used. For HER-2 detection, a semiquantitative diagnostic immunohistochemical test was used (HercepTest $^{\mathrm{TM}}$ kit, K5207; DakoCytomation). Tissue sections 
Table I. Patient and tumor characteristics and their correlation with enhanced immunoreactivity (IRS $\geq 6$ ) and nuclear-cytoplasmic expression (NCE) of PARP-1.

\begin{tabular}{|c|c|c|c|}
\hline Patient characteristics & No. $(\%)^{\mathrm{a}}$ & $\begin{array}{l}\text { PARP-1 high } \\
\text { expression }\end{array}$ & PARP-1 NCE \\
\hline All patients & $83(100)$ & & \\
\hline Age (mean $55.2 \pm 10.3 ;$ median, 55$)^{\mathrm{d}}$ & & 0.025 & 0.209 \\
\hline Menopause $^{\mathrm{d}}$ & & 0.244 & 0.339 \\
\hline Premenopausal & $27(32.5)$ & & \\
\hline Postmenopausal & $56(67.5)$ & & \\
\hline Histology: invasive ductal $\mathrm{BC}$ & $83(100)$ & & \\
\hline TNM stage according to UICC & & 0.013 & 0.236 \\
\hline II A & $33(39.8)$ & & \\
\hline II B & $50(60.2)$ & & \\
\hline Tumor size in $\mathrm{mm}(\mathrm{pT})^{\mathrm{c}}$ : mean, $31.0 \pm 12.3$; median, 30 & & 0.481 & 0.656 \\
\hline Nodal metastases $(\mathrm{N})^{\mathrm{d}}$ & & 0.221 & 0.149 \\
\hline $\mathrm{N}(-)$ & $47(56.6)$ & & \\
\hline $\mathrm{N}(+)$ & $36(43.4)$ & & \\
\hline Grading $^{\mathrm{d}}$ & & 0.003 & 0.099 \\
\hline G2 & $59(71.1)$ & & \\
\hline G3 & $24(28.9)$ & & \\
\hline \multicolumn{4}{|l|}{ Therapy ${ }^{\mathrm{b}, \mathrm{d}}$} \\
\hline Tamoxifen & $49(59.0)$ & 0.169 & 0.353 \\
\hline Cyclophosphamide/methotrexate/5-fluorouracil & $23(27.7)$ & 0.112 & 0.257 \\
\hline Anthracyclines & $1(1.2)$ & & \\
\hline Without adjuvant chemotherapy & $59(71.1)$ & & \\
\hline Radiotherapy & $37(44.6)$ & 0.081 & 0.098 \\
\hline ER status ${ }^{\mathrm{c}}$ & & 0.009 & 0.106 \\
\hline Negative & $22(26.5)$ & & \\
\hline Positive & $61(73.5)$ & & \\
\hline PgR status ${ }^{c}$ & & 0.221 & 0.230 \\
\hline Negative & $22(26.5)$ & & \\
\hline Positive & $61(73.5)$ & & \\
\hline HER-2 status ${ }^{c}$ & & 0.807 & 0.284 \\
\hline Negative $(0,1+, 2+)$ & $64(77.1)$ & & \\
\hline Positive $(3+)$ & $19(22.9)$ & & \\
\hline Recurrence $^{\mathrm{d}}$ & & 0.057 & 0.011 \\
\hline Yes & $32(38.6)$ & & \\
\hline No & $51(61.4)$ & & \\
\hline
\end{tabular}

${ }^{\mathrm{a} P e r c e n t a g e s}$ in the groups may not sum to $100 \%$ due to rounding. ${ }^{\mathrm{b} S}$ ome patients received more than one special treatment. ${ }^{\mathrm{c}} \mathrm{P}-\mathrm{value}$ of MannWhitney's U test. ${ }^{\mathrm{d}} \mathrm{P}$-value of Fisher's test. Statistically significant results $(\mathrm{P}<0.05)$ are in bold text.

were incubated with antibodies for $1 \mathrm{~h}$ at room temperature. Subsequent incubations involved biotinylated antibodies (15 min, room temperature) and a streptavidin-biotinylated peroxidase complex (15 min, room temperature) (LSAB+, HRP; DakoCytomation, Warsaw, Poland). NovaRed (Vector Laboratories, Cambridgeshire, UK) was used as a chromogen (10 min, room temperature). All sections were counterstained with Mayer's hematoxylin. In each case, control reactions were included, in which the specific antibody was substituted by a Primary Mouse Negative Control (DakoCytomation).

Evaluation of immunohistochemical reaction intensity. The intensity of the immunohistochemical reaction was estimated independently by two pathologists. In doubtful cases, a re-evaluation was performed using a double-headed microscope and staining was discussed until a consensus was reached. 
Table II. Immunocytochemical distribution of PARP-1 expression in cancer cell lines and drug-resistant sublines.

\begin{tabular}{|c|c|c|c|c|c|c|}
\hline Cell line & $\begin{array}{l}\text { Cytoplasmic PARP-1 } \\
\text { (IRS score) }\end{array}$ & $\begin{array}{l}\text { Nuclear PARP-1 } \\
\text { (IRS score) }\end{array}$ & $\begin{array}{l}\text { Origin } \\
\text { cancer }\end{array}$ & $\begin{array}{l}\text { Selection } \\
\text { agent }\end{array}$ & $\begin{array}{l}\text { Supposed resistance } \\
\text { mechanisms }\end{array}$ & Refs. \\
\hline EPP85-181P & 3 & 0 & Pancreatic & & & (24) \\
\hline EPP85-181RNOV & 6 & 6 & & Mitoxantrone & Topo II & (24) \\
\hline EPP85-181RDB & 9 & 6 & & Daunorubicin & MDR1/P-gp & (24) \\
\hline EPG85-257P & 12 & 4 & Gastric & & & $(25$ \\
\hline EPG85-257RNOV & 4 & 9 & & Mitoxantrone & $\begin{array}{l}\text { BCRP, GPC3, } \\
\text { Topo II, TAP }\end{array}$ & $(25-28)$ \\
\hline EPG85-257RDB & 4 & 4 & & Daunorubicin & MDR1/P-gp & (29) \\
\hline MCF-7 & 8 & 0 & Breast & & & $(30)$ \\
\hline MCF-7/ADR & 3 & 6 & & $\begin{array}{l}\text { Adriamycin, } \\
\text { Antiestrogens }\end{array}$ & LAMP-1 & $(31-33)$ \\
\hline
\end{tabular}

The expression of PARP-1 was evaluated using the semi-quantitative scale of the ImmunoReactive Score (IRS) according to Remmele and Stegner (23) modified by the authors (22), which took into account the percentage of reactive cells (no staining, $0 ;<25 \%, 1 ; 25-50 \%, 2 ; 51-75 \%, 3 ;>75 \%$, 4) and the intensity (no staining, 0 ; weak, 1 ; intermediate, 2 ; strong, 3) of the color reaction, with the final result being the product of both variables. Consequently, nine possible scores $(0,1,2,3,4,6,8,9$ and 12$)$ were obtained.

PARP-1 expression was only observed in the tumor compartment of BC specimens. We described two patterns of its intracellular localizations in $\mathrm{BC}$ cells: i) nuclear-cytoplasmic expression (NCE) and ii) cytoplasmic expression (CE).

Additionally, we observed that normal breast tissue, which was included in some slides, was characterized by weak to moderate nuclear-cytoplasmic PARP-1 immunoreactivity. In stromal cells and lymphocytes, nuclear and cytoplasmic PARP-1 staining was also detected.

Nevertheless, at the stage of subsequent statistical analyses, a two-grade scale system was applied, allocating 0 points for expression of PARP-1 $<6$ (low level of PARP-1 immunoreactivity) and 1 for expression of PARP-1 $\geq 6$ (high PARP-1 immunoreactivity). Definition of these two groups and determination of the cut-off point is a specific consensus of histopathological observations and statistical analyses and of the review of literature concerning PARP-1 expression evaluation.

The evaluation of estrogen and progesterone receptor expression was performed using standard methods. The staining intensity (0-3 scale) and proportion of positive cells (0-5 scale) were reported, and the Allred score that combines the two was calculated. The HER-2 status was evaluated using an FDA-approved scoring system of $0,1+, 2+$ and $3+$ ( 0 , no immunostaining; $1+$, weak immunostaining, $<30 \%$ of the tumor cells; $2+$, complete membranous reactivity, either uniform or weak in at least $10 \%$ of the tumor cells; $3+$, uniform intense membranous staining in at least $30 \%$ of the tumor cells).

Cell lines. In the present study, we used 4 human $\mathrm{BC}$ cell lines: MCF-7, CAMA-1, SK-BR-3 and R-103 and 3 models of drug-resistant cell lines. Characteristics and culture of the human gastric carcinoma cell line EPG85-257P (257P), human pancreatic cell line EPP85-181P (181P) and human BC cell line (MCF-7), its classical MDR variants EPG85-257RDB (257RDB) and EPP85-181RDB (181RDB) overexpressing MDR1/P-gp, and its atypical MDR variants EPG85-257RNOV (257RNOV), EPP85-181RNOV (181RNOV), MCF-7/ADR were previously described in detail (Table II) (24-33).

Cell culture. Cells were grown in Leibovitz L-15 medium (BioWhittaker Inc., Walkersville, MD, USA) supplemented with $10 \%$ fetal calf serum (FCS; Gibco/BRL, Grand Island, NY, USA), $1 \mathrm{mM}$ L-glutamine, $6.25 \mathrm{mg} / 1$ fetuin, $80 \mathrm{IE} / 1$ insulin, $2.5 \mathrm{mg} / \mathrm{ml}$ transferrin, $0.5 \mathrm{~g} / 1$ glucose, $1.1 \mathrm{~g} / 1 \mathrm{NaHCO}_{3}$, $1 \%$ minimal essential vitamins and 20,000 kIE/l trasylol in a humidified atmosphere of $5 \% \mathrm{CO}_{2}$ at $37^{\circ} \mathrm{C}$. Prior to resistance testing, Mycoplasma tests were performed using the Venor Mp kit, according to the manufacturer's instructions (Minerva Biolabs GmbH, Berlin, Germany).

Resistance tests. Drugs were used in their commercially available form (except cyclophosphamide, which was used in its activated form). Each drug was applied to the cells in 3 concentrations $(\mathrm{C} 1, \mathrm{C} 2$ and $\mathrm{C} 3) . \mathrm{C} 1=10^{-1} \times \mathrm{C} 2$ and $\mathrm{C} 3=10 \times \mathrm{C} 2$. Concentration $\mathrm{C} 2$ (the clinically available drug in the tumor) was deduced from levels assessed to be clinically achievable in tumor tissue, as previously discussed (Table III) (30).

In each experiment, 500 cells/microtiter dish were seeded onto 96-well plates. After 2 days, precontrol cells were fixed and stained using sulforhodamine B (SRB). At the same time, triplicate cultures were prepared with all 11 studied drugs at $\mathrm{C} 1, \mathrm{C} 2$ and $\mathrm{C} 3$ concentrations. After 4 days, incubation was terminated by replacing the medium with $10 \%$ trichloroacetic acid, followed by incubation at $40^{\circ} \mathrm{C}$ for $1 \mathrm{~h}$. Subsequently, the plates were washed 5 times with water and stained by adding $100 \mu 10.4 \%$ SRB (Sigma, St. Louis, MO, USA) in $1 \%$ acetic acid for $10 \mathrm{~min}$ at room temperature. Washing the plates 5 times with $1 \%$ acetic acid eliminated unbound dye. After air-drying and resolubilization of the protein-bound dye in $10 \mathrm{mM}$ Tris- $\mathrm{HCl}(\mathrm{pH} 8.0)$, absorbance was read at $562 \mathrm{~nm}$ in an Elisa-Reader (EL 340 Microplate Bio Kinetics Reader; 
Table III. Drugs used to establish resistance patterns and their $\mathrm{C} 2$ concentrations (the clinically available drug in the tumor).

\begin{tabular}{lcl}
\hline Drug & $\mathrm{C} 2(\mu \mathrm{M} / \mathrm{ml})$ & \multicolumn{1}{c}{ Supplier } \\
\hline 5-Fluorouracil & $38.43 \times 10^{-5}$ & Gry-Pharma \\
Cisplatin & $16.66 \times 10^{-5}$ & Gry-Pharma \\
Cyclophosphamide & $50.16 \times 10^{-5}$ & Asta Werke \\
(hydroxylated) & & \\
Doxorubicin & $0.86 \times 10^{-5}$ & Cell-Pharma \\
Etoposide & $2.37 \times 10^{-5}$ & Gry-Pharma \\
Methotrexate & $0.3 \times 10^{-5}$ & Wyeth-Lederle \\
Mitomycin C & $1.49 \times 10^{-5}$ & Hexal \\
Mitoxantrone & $0.38 \times 10^{-5}$ & Wyeth-Lederle \\
Paclitaxel & $0.29 \times 10^{-5}$ & Bristol \\
Topotecan & $\times 10^{-5}$ & GlaxoSmithKline \\
Vinblastine & $0.1 \times 10^{-5}$ & Gry-Pharma \\
\end{tabular}

Bio-Tek Instruments, Winooski, VT, USA). The measurements were performed in triplicate in 3 independent experiments. For the calculation of the RI values, the averages of all 9 measurements were used. The resistance index (RI) was estimated by the formula: $\mathrm{RI}=\left(\mathrm{n}_{\text {post }} / \mathrm{n}_{\text {pre }}\right) \times\left[\left(\mathrm{n}_{2}-\mathrm{n}_{\text {pre }}\right) /\left(\mathrm{n}_{\text {post }}-\mathrm{n}_{\text {pre }}\right) \times 100\right]$ where $\mathrm{n}_{\text {pre }}$ is the medium absorbance value of precontrol at the C2 concentration, $\mathrm{n}_{\text {post }}$ is the medium absorbance value of control and $n_{2}$ is the medium absorbance value of stained cells tested with the chosen concentration of the studied drug.

Immunocytochemistry. Immunostaining of PARP-1 was performed using the studied panel of BC cell lines. Cells were grown on microscopic slides and fixed in ice-cold methanolacetone mixture $(1: 1)$ for $10 \mathrm{~min}$. Immunostaining reaction was performed in triplicate as previously described.

Statistical analysis. Statistical analysis was performed using the Statistica 9.1 software package (StatSoft Inc., Tulsa, OK, USA). OS was defined as the time between the primary surgical treatment and mortality, and OS was censored at last follow up for those who were alive. DFS was defined as the time between the primary surgical treatment and date of relapse or mortality, whichever occurred first. DFS was censored at the last followup for patients who survived without disease recurrence. CSOS was defined as the time between the primary surgical treatment and cancer-associated mortality, and was censored at the last follow-up for surviving patients.

Due to the important role of nodal metastatic tumors as negative prognostic factors, an additional analysis of the role of PARP-1 expression and its subcellular localization in patients with and without regional lymph node metastasis (N+ and $\mathrm{N}-)$ was performed.

The $\chi^{2}$ test, exact Fisher test in case of $2 \times 2$ tables and Kendall rank correlation were used to analyze associations between PARP-1 protein expression parameters and clinicopathological parameters. Differences between two groups were tested with the Mann-Whitney U test, the log-rank test was used to compare survival in two groups, the OS rate was estimated by the Kaplan-Meier method and the influence of explanatory variables on mortality risk was analyzed by means
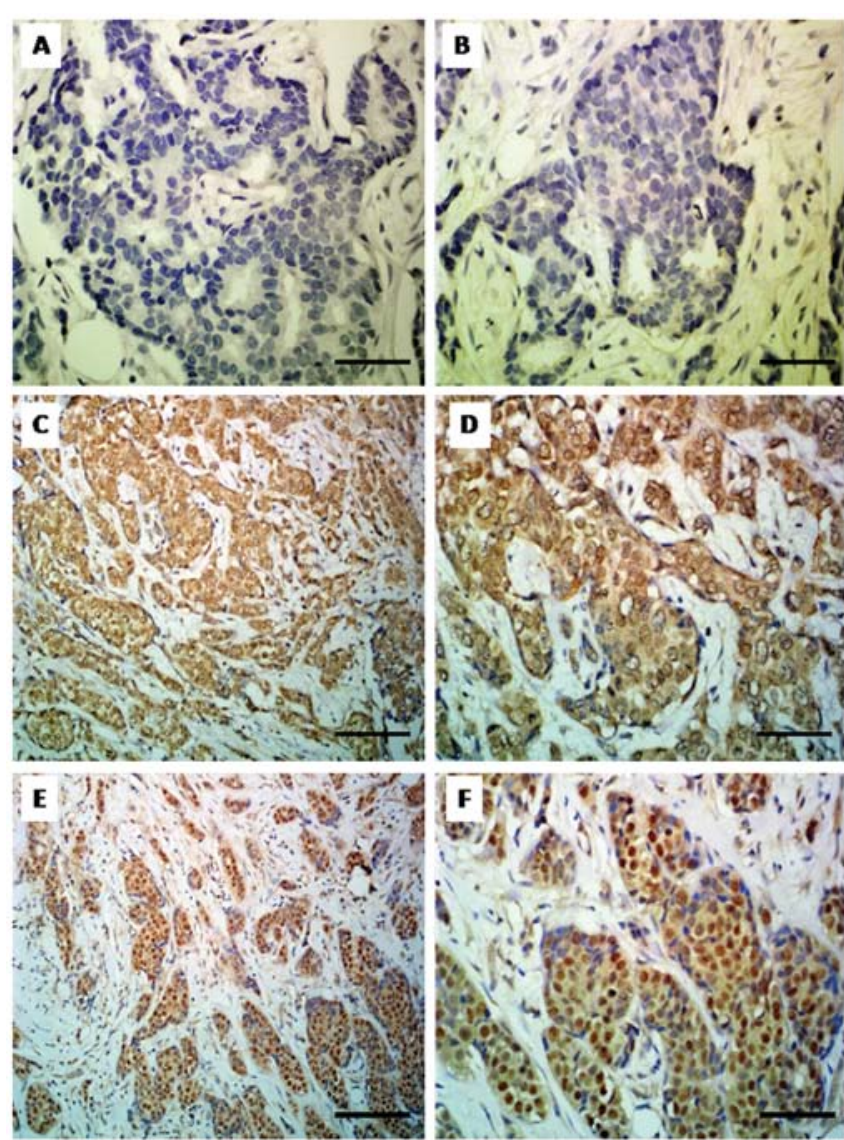

Figure 1. Immunohistochemical analysis of PARP-1 expression. (A and B) Lack of PARP-1 expression in breast cancer cells [ImmunoReactive Score (IRS) 0, x400, hematoxylin]. (C) Cytoplasmic expression of PARP-1 in breast cancer cells (IRS 8; magnification, x200; hematoxylin). (D) Cytoplasmic expression of PARP-1 (IRS 8; magnification, x400; hematoxylin). (E) Nuclearcytoplasmic expression (NCE) of PARP-1 (IRS 12; magnification, x200; hematoxylin). (F) NCE of PARP-1 (IRS 12; magnification, x400; hematoxylin). Bars: A, B, D and F, $50 \mu \mathrm{m}$; C and E, $100 \mu \mathrm{m}$.

of the Cox proportional hazard regression and logistic regression in case of binary survival. P-values $<0.05$ were considered to indicate statistically significant differences.

\section{Results}

PARP-1 immunostaining in BC specimens. PARP-1 expression defined as IRS $>0$ was found in the entire group of 83 patients subjected to investigation. The average IRS was $6.48 \pm 2.5$ and the median was 8 . For the purposes of statistical analysis, enhanced immunoreactivity of PARP-1 was defined as IRS $\geq 6$ (55 patients, $66.27 \%$ ), while low immunoreactivity was assigned IRS values between 0 and 4 (28 patients, 33.73\%) (Fig. 1A and B). Histopathological evaluation of the specimens revealed two patterns of PARP-1's subcellular localizations. Cytoplasmic localization alone was observed in 48 cases $(57.83 \%)$ (Fig. 1C and D), whereas nuclear-cytoplasmic localization was identified in 35 cases (42.17\%) (Fig. 1E and F).

Relationship between PARP-1 expression and status of steroid receptors and HER-2 reactivity. There was a statistically significant correlation between overexpression of PARP-1 (IRS $\geq 6$ ) and positive ER status $(\mathrm{P}=0.009)$. No significant 
Table IV. Univariate analysis of correlations between immunohistochemical parameters of PARP-1 expression and 5-, 10- and 15-year overall survival and multivariable Cox regression analysis of PARP-1 expression and 15-year cancer-specific overall survival in the group without lymph node metastases, with lymph node metastases and in the whole cohort of patients.

Parameters of

Univariate logistic regression

\begin{tabular}{|c|c|c|c|c|}
\hline PARP-1 expression & 5-year survival ${ }^{\mathrm{a}}$ & 10-year survival ${ }^{\mathrm{a}}$ & 15 -year survival ${ }^{\mathrm{a}}$ & 15-year survival Odds Ratio (95\% CI) \\
\hline$\%$ of positive cells & 0.285 & 0.129 & 0.037 & $2.00(0.98-4.06)$ \\
\hline Intensity & 0.684 & 0.197 & 0.029 & $2.32(1.05-5.17)$ \\
\hline IRS & 0.399 & 0.087 & 0.006 & $1.30(1.06-1.58)$ \\
\hline High expression (IRS $\geq 6$ ) & 0.270 & 0.464 & 0.039 & $2.79(1.00-7.75)$ \\
\hline $\mathrm{NCE}$ & 0.517 & 0.095 & 0.015 & $3.08(1.21-7.83)$ \\
\hline
\end{tabular}

Multivariable Cox regression analysis of 15-year survival

\begin{tabular}{|c|c|c|c|c|c|c|}
\hline \multirow[b]{2}{*}{ Clinicopathological parameters } & \multicolumn{2}{|c|}{ All patients } & \multicolumn{2}{|c|}{$\begin{array}{l}\text { With lymph node } \\
\text { metastases }(\mathrm{N}+)\end{array}$} & \multicolumn{2}{|c|}{$\begin{array}{l}\text { Without nodal } \\
\text { metastases (N-) }\end{array}$} \\
\hline & P-value & $\operatorname{HR}(95 \% \mathrm{CI})$ & P-value & $\mathrm{HR}(95 \% \mathrm{CI})$ & P-value & $\operatorname{HR}(95 \% \mathrm{CI})$ \\
\hline Tumor size (pT) & 0.026 & $1.05(1.01-1.09)$ & 0.170 & $1.04(0.98-1.09)$ & 0.036 & $1.06(1.00-1.12)$ \\
\hline NCE & 0.016 & $2.68(1.21-5.98)$ & 0.092 & $2.29(0.87-6.01)$ & $\mathbf{0 . 0 3 3}$ & $8.88(1.19-66.29)$ \\
\hline$\%$ of PARP-1 positive cells ${ }^{\mathrm{b}}$ & 0.028 & $2.81(1.12-7.03)$ & 0.033 & $3.68(1.11-12.20)$ & 0.756 & $0.77(0.14-4.13)$ \\
\hline Nodal metastases & 0.0003 & $4.79(2.04-11.23)$ & - & - & - & - \\
\hline
\end{tabular}

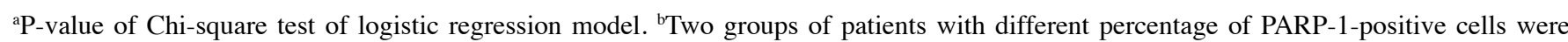
analyzed: $0-75$ vs. $>75 \%$. Statistically significant results $(\mathrm{P}<0.05)$ are in bold text. NCE, nuclear-cytoplasmic expression.

correlations were identified for PgR or HER-2 status (Table I). Intracellular localization of PARP-1 [nuclear-cytoplasmic (NCE); cytoplasmic (CE)] showed no statistically significant correlations with ER, PgR or HER-2 expression parameters.

Relationship between PARP-1 expression and clinicopathological parameters. Higher tumor grading $(G)$ showed statistically significant correlation with low expression of PARP-1, defined as IRS 0-4 ( $\mathrm{P}=0.003)$. Paradoxically, PARP-1 overexpression was closely related to higher stage according to UICC (II B vs. II A) (P=0.013). Furthermore, PARP-1 overexpression was significantly more frequent in patients who were older at the time of diagnosis $(\mathrm{P}=0.025)$. Additionally, higher probability of cancer recurrence was noted in a group of patients with PARP-1 overexpression, albeit at the limit of statistical significance (PARP-1 overexpression was identified in $78.13 \%$ patients with cancer; $\mathrm{P}=0.057$ ) (Table I).

No statistically significant correlations were observed between overexpression of PARP-1 and the size of the tumor, the presence of lymph node metastases, menopausal status or the type of adjuvant therapy (Table I).

Additionally it was shown that subcellular localization of PARP-1 is key for the recurrence of BC. In patients with nuclear-cytoplasmic (NCE) topography, the recurrence of cancer was highly probable, especially in lung $(\mathrm{P}=0.011)$ (Table I).

PARP-1 immunoreactivity and patient survival; 5-year, 10-year and 15-year observation. No statistically significant correlations were identified between overexpression of PARP-1 and its localization as regards 5- and 10- year OS; however 15-year observations presented notable results. The initial non-significant tendency for higher mortality risk observed within the first 10 years after diagnosis augmented within the following 5-year observation period (10th-15th year) and the analysis of 15-year survival rates showed that overexpression of PARP-1 (IRS $\geq 6$ ) was a statistically significant, unfavorable prognostic factor. Almost $80 \%$ of patients with PARP-1 overexpression died during the 15 -year observation period $(\mathrm{P}=0.039)$ (Table IV). It is worth noting, that nuclear-cytoplasmic localization also proved to be an unfavorable prognostic factor $(\mathrm{P}=0.015)$ only during the 15 -year observation period; only $30 \%$ of patients with nuclear-cytoplasmic immunotopography survived the 15 -year long observation period. Similar to the case of other parameters of expression of the protein under study, the initially non-significant difference in its immunotopography became a strong prognostic factor after 10 years of observation (Table IV).

The Kaplan-Meier estimators also confirmed the findings. Nuclear-cytoplasmic localization was closely correlated with unfavorable prognosis as compared with patients in whom cytoplasmic of PARP-1 topography $(\mathrm{P}=0.020)$ alone was identified (Fig. 2B). Patients with overexpression of PARP-1 defined as IRS $\geq 6$ were found to have a tendency of lower survival rate (Fig. 2A) during the 15-year clinical observation period as compared with the patients with low expression of PARP-1 ( $\mathrm{P}=0.059)$.

Prognostic significance of PARP-1 expression in lymph node negative $(N-)$ and lymph node positive $(N+)$ patients. Nodal 
A
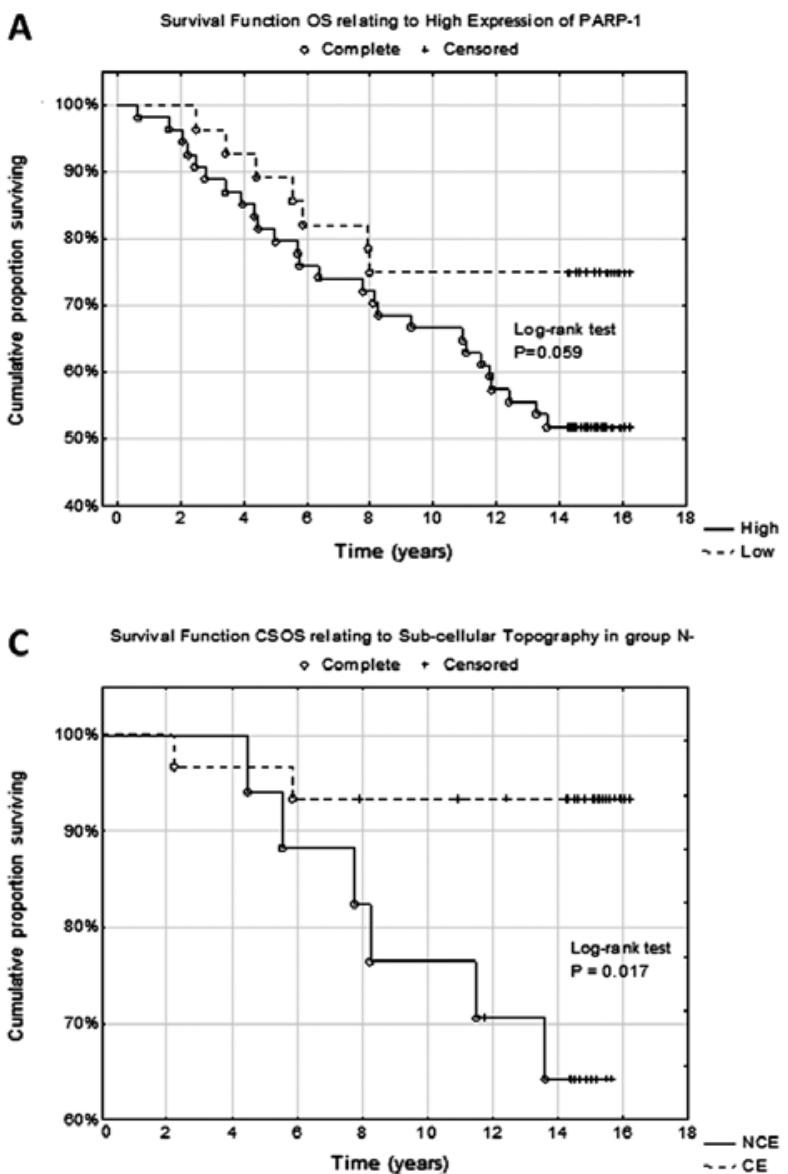

E

Sunival Function CSOS relating to Sub-cellulat Topography in group $\mathrm{N}^{+}$ - Complete + Censored

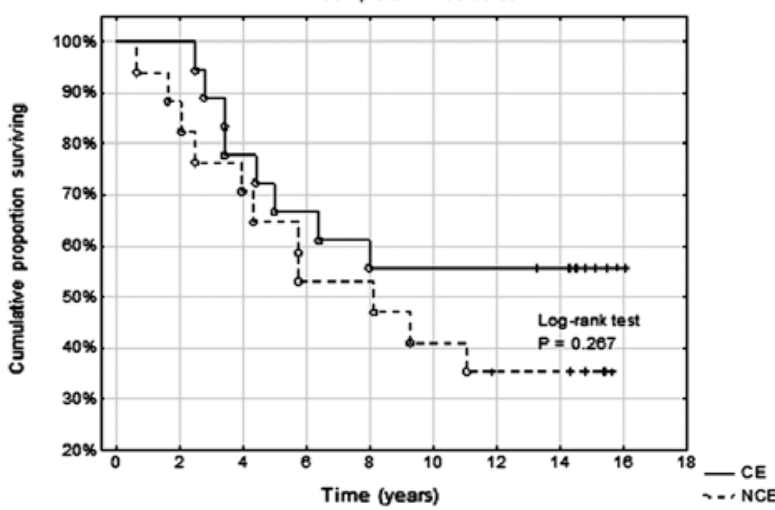

B

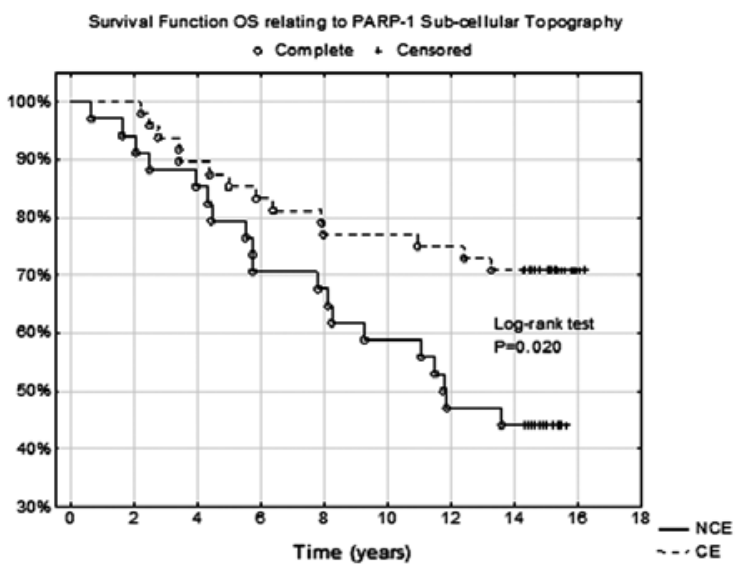

D

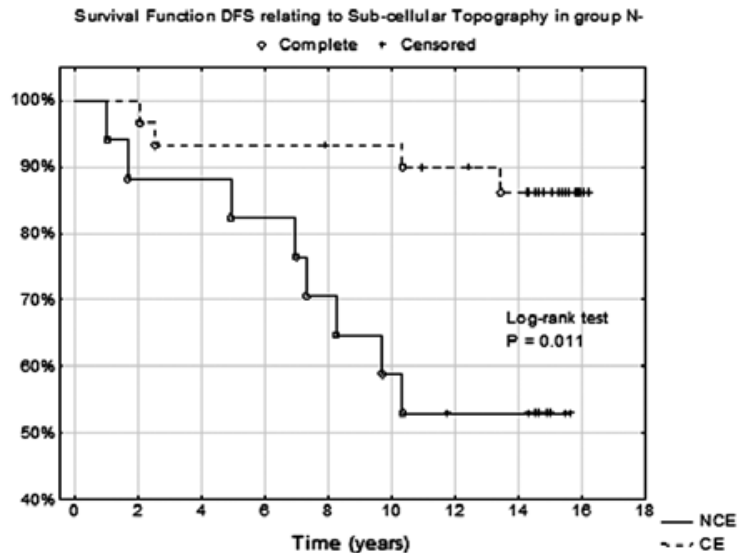

$\mathbf{F}$ Sunvival Function DFS rela ting to Sub-cellular Topogra phy in group $\mathrm{N}+$ - Complete + Censored

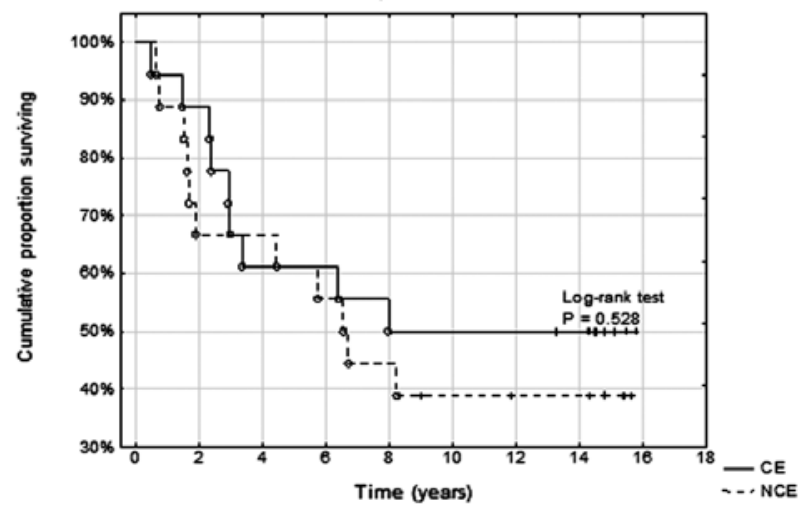

Figure 2. (A) Strong PARP-1 expression defined as IRS $\geq 6$ is related ( $P=0.059$ ) to shorter overall survival (OS) (solid line) during the 15 -year clinical observation period as compared with low expression patients (dotted line). (B) Nuclear-cytoplasmic expression (NCE) was significantly correlated with poor prognosis as compared with patients in whom cytoplasmic topography (CE) of PARP-1 (P=0.020) alone was identified. Comparison of prognostic significance of NCE of PARP-1 in regional lymph-node negative (N-) patients (C and D) and in lymph-node positive (N+) patients (E and F) in cancer-specific OS and disease-free survival analysis. (C and D) Nuclear-cytoplasmic localization is a statistically significant, unfavorable prognostic factor only in regional $\mathrm{N}$ - patients. (E and F) No significant correlations were identified between nuclear-cytoplasmic topography and N+ patient survival.

metastases identified in histopathological examination are a strongly unfavorable prognostic factor in the analyzed group of patients $(\mathrm{P}<0.001)$, which justified the necessity to perform an additional analysis of PARP-1 expression in both subgroups of patients $(\mathrm{N}$ - and $\mathrm{N}+$ ). It was found that only in $\mathrm{N}$ - patients nuclear-cytoplasmic localization was an unfavorable prognostic factor exclusively in CSOS and DFS analysis $(\mathrm{P}=0.017$, $\mathrm{P}=0.011$, respectively) (Fig. 2C and D). No significant correlation was demonstrated for NCE of PARP-1 during OS analysis
$(\mathrm{P}=0.074)$. It should be noted that the type of subcellular distribution of PARP-1 had no prognostic value in $\mathrm{N}+$ patients (Fig. 2E and F). No other statistically significant correlations related to the prognostic significance of overexpression or individual parameters of PARP-1 immunoreactivity in $\mathrm{N}$ - or $\mathrm{N}+$ patients.

Multivariable Cox regression analysis. The following four parameters (Table IV), which in the multivariate proportional 

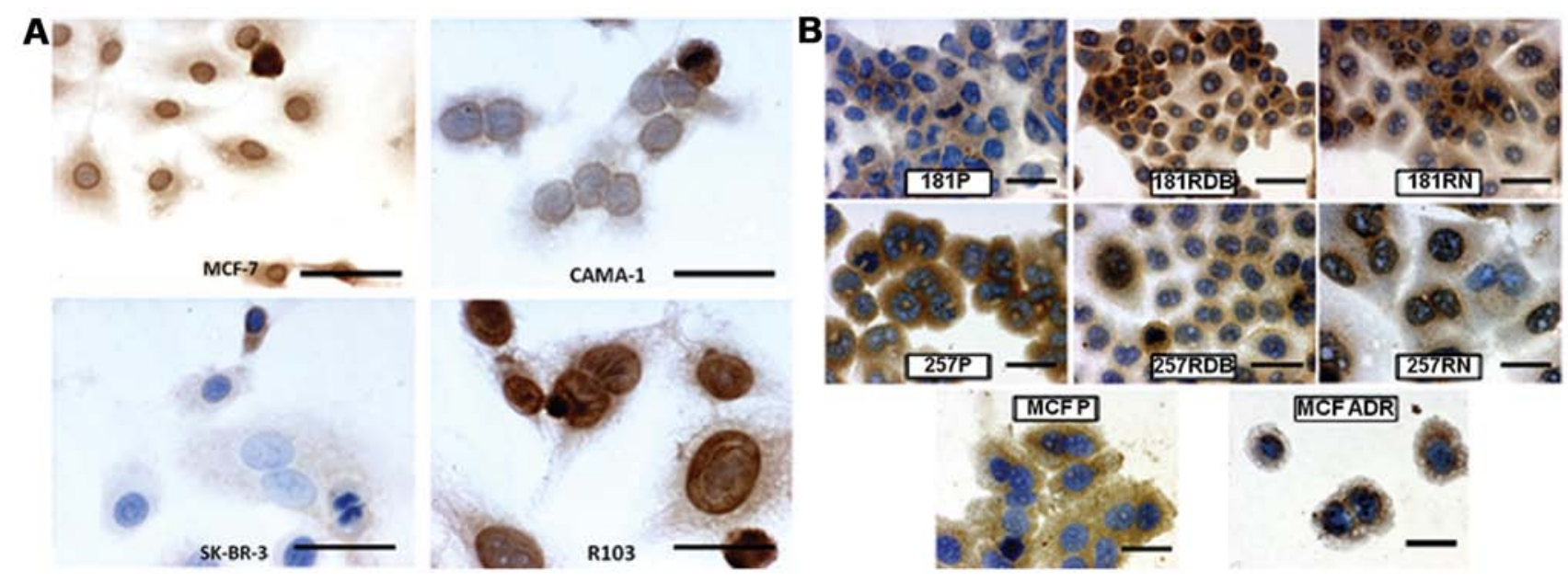

Figure 3. (A) Immunocytochemical localization of PARP-1 expression in cells of the MCF-7, CAMA-1, SK-BR-3 and R-103 breast cancer cell lines (x400, hematoxylin). (B) Immunocytochemical localization of PARP-1 expression in cells of EPG85-257P (257P), EPG85-257RDB (257RDB), EPG85-257RNOV (257RNOV), EPP85-181P (181P), EPP85-181RDB (181RDB), EPP85-181RNOV (191RNOV), MCF-7 and MCF-7/ADR (x600, hematoxylin). Bars: A, 12,5 $\mu$ m; $\mathrm{B}, 6.25 \mu \mathrm{m}$.

hazard regression model with backward stepwise variables elimination proved to have a statistically significant effect on the prognosis, were found to be independent factors of poor prognosis in the group of patients subjected to the study: i) occurrence of metastasis in lymph nodes $(\mathrm{P}=0.0003)$, ii) tumor size $(\mathrm{P}=0.026)$, iii) nuclear-cytoplasmic localization of PARP-1 ( $\mathrm{P}=0.016)$, and iv) high percentage ( $0-75$ vs. $>75 \%$ positive cells) of cells with PARP-1 expression ( $\mathrm{P}=0.028)$. Other clinicopathological parameters did not significantly influence the prognosis in the multivariable Cox regression analysis.

Due to a decisive influence of lymph node metastases on prognosis, multivariate analysis was conducted separately in $\mathrm{N}-$ and $\mathrm{N}+$ groups of patients (Table IV). It was demonstrated that for N- patients, nuclear-cytoplasmic topography of PARP-1 was an independent unfavorable prognostic factor $(\mathrm{P}=0.033)$, which confirmed earlier findings of univariate analysis. Additionally, in $\mathrm{N}$ - patients, it was found that the larger the tumor the poorer the prognosis was $(\mathrm{P}=0.035)$.

In N+ patients, high percentage of PARP-1-positive cells had a significant, unfavorable influence on poor outcome in the 15-year observation $(\mathrm{P}=0.033)$. Other clinicopathological factors did not show statistically significant prognostic value in the multivariate analysis.

PARP-1 expression in relation to drug-resistance. The studied $\mathrm{BC}$ cell lines demonstrated the following intensity of reaction for PARP-1: MCF-7, cytoplasmic PARP-1, IRS=4; nuclear PARP-1, IRS=8; CAMA-1, cytoplasmic PARP-1, IRS=2; nuclear PARP-1, IRS=4; SK-BR-3, cytoplasmic PARP-1, IRS=3; nuclear PARP-1, IRS=1; R-103, cytoplasmic PARP-1, IRS $=3$; nuclear PARP-1, IRS $=12$. The results of conducted cytotoxicity tests and the results of immunocytochemical reactions are depicted in Figs. 3A and 4. The investigations demonstrated no relationship between expression of PARP-1 in BC cells and sensitivity of the cells to cytostatic drugs.

In the case of cytostatic-resistant cell line models, we revealed varying positive immunocytochemical reactions. Cytoplasmic localization of PARP-1 did not correlate with sensitivity to cytotoxic drugs, but we observed a significantly higher intensity of PARP-1 immunoreaction in the nucleus of the cytostatic-resistant cell lines (Table II, Fig. 3B).

\section{Discussion}

In the present study, we investigated the expression of PARP-1 in a homogeneous group of patients with stage II invasive ductal BC. In addition, we assessed relationships between the subcellular localization of this protein, clinicopathological parameters and patient survival over a 15 -year period. In vitro analysis demonstrated no relationship between expression of PARP-1 in BC cells and sensitivity of the cells to 11 cytostatic drugs. Notably, in the case of cytostatic-resistant cell line models, we revealed that the cytoplasmic localization of PARP-1 did not correlate with sensitivity to cytotoxic drugs, but we observed a significantly higher intensity of PARP-1 immunoreaction in the nucleus of the cytostatic-resistant cell lines. Further extensive studies are required to describe the part played by a specific protein in cytotoxic drug resistance. In the present study, we suggested only that heightened expression of PARP-1 is a characteristic for cells resistant to cytotoxic drugs. This phenomenon might only be a phenotype. However, the basis of the studies on the new predictive markers is to find phenotypes with characteristic patterns for different drug resistance.

In the present study, overexpression of PARP-1 defined as IRS $\geq 6$ was found in $66.3 \%$ of patients (55 patients), while low immunoreactivity of PARP-1 was observed in $33.7 \%$ (28 patients). Cytoplasmic localization alone was observed in 48 cases (57.83\%), and nuclear-cytoplasmic localization was observed in 35 cases $(42.17 \%)$. Domagala et al (34) with the use of tissue microarray, immunohistochemically determined the expression of PARP-1 in 130 cases of BRCA1-dependent $\mathrm{BC}$ and in 594 cases of sporadic BC (BRCA1-non-related). Using the QS index (QuickScore Method), high percentage of BC with overexpression of PARP-1 was identified since enhanced reactivity of PARP-1 was noted in as many as $81.5 \%$ of cases (106/130) of patients with BRCA1-dependent BC and 

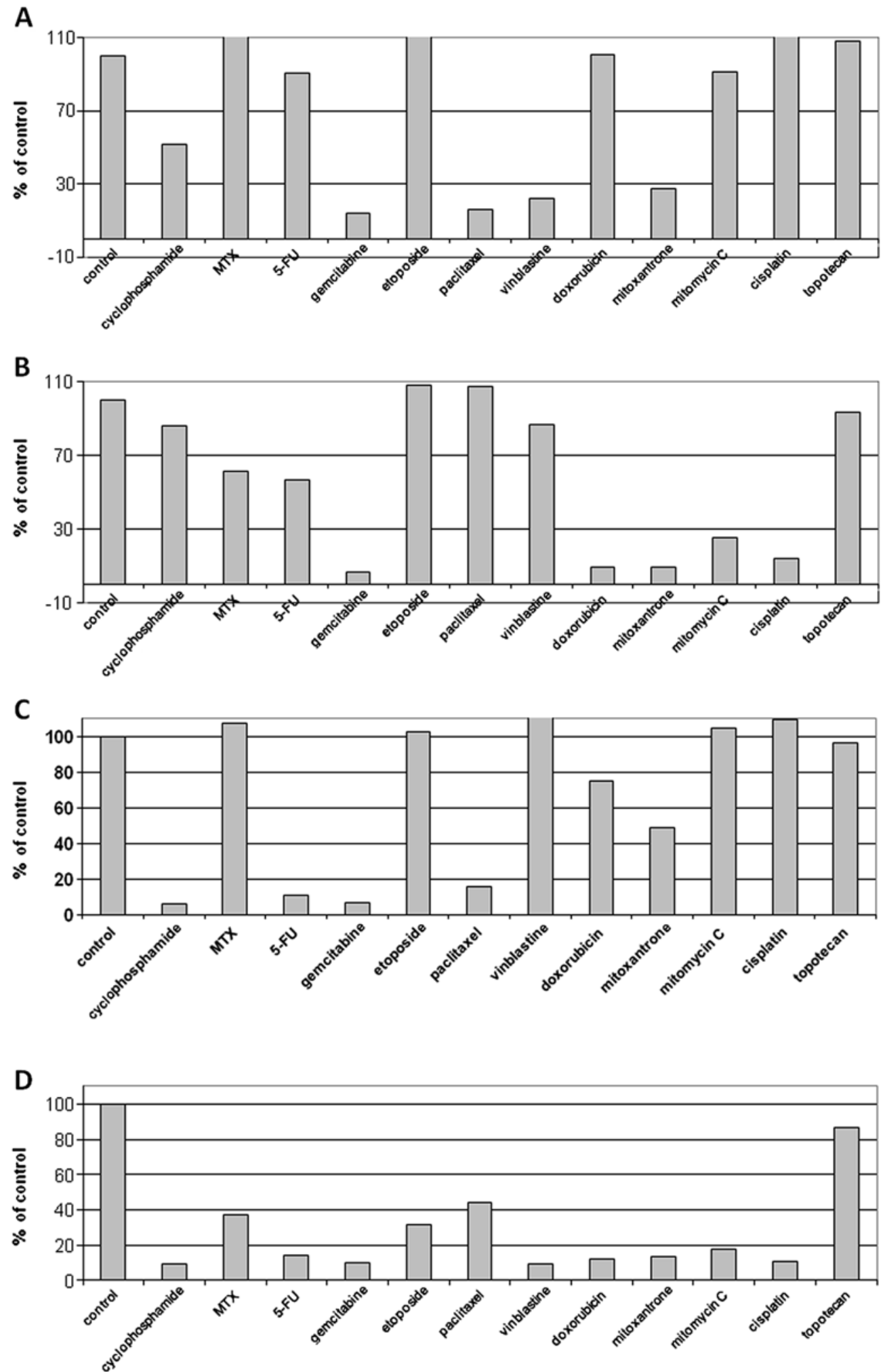

Figure 4. Sensitivity of the studied cells to 11 cytostatic drugs of various groups. (A) MCF-7 breast cancer cell line, (B) CAMA-1 breast cancer cell line, (C) SK-BR-3 breast cancer cell line and (D) R-103 breast cancer cell line.

in as many as $91.2 \%(542 / 594)$ with BRCA1-independent BC (34). Furthermore, in the analyzed publication, the dominant subcellular localization of PARP-1 was nuclear expression alone $(86.2 \%, 112 / 130$ BRCA1-dependent BC; 93.8\%, 557/594 BRCA1-independent BC). von Minckwitz et al (35) showed overexpression of PARP-1 in only $23.7 \%$ of BC cases (151/638 patients); however, their study covered significantly wider clinical and histopathological spectrum of BCs (ductal and lobular histological type; G1-3; cT2-4) as compared with the present study. Similar to our observations, von Minckwitz et al (35) confirmed the presence of PARP-1 within two cellular compartments, namely cytoplasmic and nuclear ones. 
Markedly, nuclear localization was the only one observed by Rojo et al (36) conducting research into overexpression of PARP-1 in 330 cases of BC. The results may be due to a very specific method of measuring overexpression of PARP-1. Computer assisted microscopic image analysis was used and the optical density (OD) was calculated. OD values ranged from 29 to 133.094 , and 39.970 proved to be a limit value over which overexpression of PARP-1 was defined. With this definition of cut-off point, enhanced reactivity of PARP-1 was found in $31.2 \%$ cases of BC (36).

In the present study, statistical analysis showed that enhanced PARP-1 expression is closely correlated with positive ER status $(\mathrm{P}=0.009)$. No statistically significant correlations were identified between PARP-1 expression and PgR or HER-2 status.

Similar results as regards the lack of correlation of PARP-1 overexpression with PgR or HER-2 status were obtained by Rojo et al (36), although it must be stressed that in the study, a strong significant correlation between positive PARP-1 status and the lack of ER expression was identified. Results similar to those of Rojo et al (36) discussed above were reported by von Minckwitz et al (35) who also showed significant correlation between PARP-1 overexpression and negative ER and PgR status, but, similar to our results, found no significant correlations with HER-2 overexpression. Ozretic et al (37) investigated the correlation between increased PARP-1 reactivity in BC cells and negative ER and PgR status. The researchers showed that PARP-1 overexpression in nuclear localization is closely correlated with positive ER and PgR status which corresponds to our findings and rejects the hypothesis that overexpression of PARP-1 is restricted to triple negative BC phenotype.

No correlation between PARP-1 overexpression and lymph node metastases was found. However, von Minckwitz et al (35) demonstrated that high reactivity of PARP-1 in cancer tissue is correlated with the occurrence of metastases in regional lymph nodes. Other researchers did not show statistically significant correlations between increased PARP-1 immunoreactivity and the occurrence of nodal metastases (36).

In the present study, higher tumor grading (G) showed statistically significant correlation with low PARP-1 expression $(\mathrm{P}=0.003)$. It is an unexpected result which confirms that the role of PARP-1 in BC biology needs to be studied further as two independent research groups have shown that high tumor grade is closely correlated with PARP-1 overexpression $(35,36)$. Dual role of PARP-1 overexpression is confirmed in another correlation that indicates that PARP-1 overexpression is closely related to a more advanced clinical stage according to UICC (II B vs. II A) ( $\mathrm{P}=0.013)$. Furthermore, PARP-1 overexpression was significantly more frequent in patients who were older at diagnosis $(\mathrm{P}=0.025)$. No statistically significant correlations were identified between PARP-1 overexpression and tumor size, which is consistent with the observation of von Minckwitz et al (35) and Rojo et al (36).

The present study showed that subcellular localization of PARP-1 is of key importance for the recurrence of $\mathrm{BC}$, especially in lung. Since Rojo et al (36) described only nuclear localization of PARP-1, all relations associated with negative ER and PgR status, grade (high G) and adverse prognosis are valid for this sublocalization. In the study by von Minckwitz et al (35), nuclear localization was of no importance both as regards prognosis and analysis with clinicopathological parameters.

The key stages of the research were analyses of PARP-1 expression as regards its prognostic value for 5-, 10- and 15 -year survival rates. The results were notable as no statistically significant correlations were found between PARP-1 overexpression and its localization as regards 5- and 10-year survival; however, the 15-year observation presented marked results. It was found that PARP-1 overexpression (IRS $\geq 6$ ) was a significant, adverse prognostic factor only after 10-15 years of the initial diagnosis $(\mathrm{P}=0.039)$. It should be noted that the nuclear-cytoplasmic localization also proved to be an adverse prognostic factor only in the course of the 15 -year observation period $(\mathrm{P}=0.015)$.

Nuclear-cytoplasmic localization of PARP-1 expression had an adverse effect on the prognosis for lymph node negative $(\mathrm{N}-)$ patients $(\mathrm{P}=0.011)$. No such correlation was found for lymph node positive $(\mathrm{N}+)$ patients. Therefore, $\mathrm{NCE}$ became a marker of distant recurrence in patients with potentially favorable prognosis.

Von Minckwitz et al (35) showed a significant correlation between shorter DFS and shorter OS and PARP-1 overexpression. However, in the present study, cytoplasmic localization was an unfavorable prognostic and no significant correlations were observed as regards PARP-1 nuclear expression. Similar to our results, Rojo et al (36) showed that PARP-1 overexpression and its nuclear localization are statistically significant unfavorable prognostic factors that are closely related to very high risk of cancer recurrence and cancer-specific mortality in the course of $\mathrm{BC}$ disease.

We acknowledge that the small patient population is a limitation of the present study. However, the number of patients was strongly influenced by the single series performed by our institution and highly homogenous characteristic of breast tumors (ductal invasive BC, G2 and G3, clinical stage II according to UICC, Madden mastectomy). Indeed, such a long follow-up period is rare and, in this regard, our research group is unique. Evaluation of stage II ductal BC patients eliminated part of the clinical variables that could bias the analysis of prognostic significance. It should also be emphasized that our patient cohort is well described and researched. The results of many analyses performed on this group (originally larger, approximately 100 cases, currently only 85 due to the usage of tissue material) were previously published (38-40).

The critical role of the long observation period of $\mathrm{BC}$ patients must be stressed since only 10 years after the initial diagnosis did the differences in survival of analyzed subgroups of patients appear and the prognostic significance of PARP-1 overexpression and its subcellular localization could be analyzed. This finding is of note as no study published until October 2013 described a group of patients subjected to such a long observation. Considering the continuous improvement of BC therapy, a several year observation that is significantly longer than the most frequently reported 5-year observation period is crucial for the evaluation of the prognostic value of PARP-1 expression.

In conclusion, in non-advanced and $\mathrm{N}-\mathrm{BC}$ patients who are classified as having favorable prognosis, a subgroup with a potentially poorer long-term prognosis (shorter OS, CSOS and DFS) was identified. Nuclear-cytoplasmic localization of 
PARP-1 expression was more frequently identified in these patients. This correlation provides a notable basis for further studies; however, it remains to be confirmed in larger cohort studies.

\section{Acknowledgements}

This study was supported by Wroclaw Medical University research grant ST-593.

\section{References}

1. Goldhirsch A, Wood WC, Coates AS, Gelber RD, Thürlimann B, Senn HJ and Panel members: Strategies for subtypes - dealing with the diversity of breast cancer: highlights of the St Gallen International Expert Consensus on the Primary Therapy of Early Breast Cancer 2011. Ann Oncol 22: 1736-1747, 2011.

2. Chambon P, Weill JD and Mandel P: Nicotinamide mononucleotide activation of new DNA-dependent polyadenylic acid synthesizing nuclear enzyme. Biochem Biophys Res Commun 11: $39-43,1963$

3. Kiliańska ZM, Żołnierczyk J and Węsierska-Gądek J: Biological activity of poly(ADP-ribose) polymerase-1. Postepy Hig Med Dosw 64: 344-363, 2010 (In Polish).

4. Shieh WM, Ame JC, Wilson MV, Wang ZQ, Koh DW, Jacobson MK and Jacobson EL: Poly(ADP-ribose) polymerase null mouse cells synthesize ADP-ribose polymers. J Biol Chem 273: 30069-30072, 1998.

5. Otto H, Reche PA, Bazan F, Dittmar K, Haag F and Koch-Nolte F: In silico characterization of the family of PARP-like poly(ADPribosyl)transferases (pARTs). BMC Genomics 6: 139, 2005.

6. Krishnakumar R and Kraus WL: The PARP side of the nucleus: molecular actions, physiological outcomes, and clinical targets. Mol Cell 39: 8-24, 2010.

7. Mangerich A and Bürkle A: How to kill tumor cells with inhibitors of poly(ADP-ribosyl)ation. Int J Cancer 128: 251-265, 2011.

8. Langelier MF, Servent KM, Rogers EE and Pascal JM: A third zinc-binding domain of human poly(ADP-ribose) polymerase-1 coordinates DNA-dependent enzyme activation. J Biol Chem 283: 4105-4114, 2008

9. Langelier MF, Ruhl DD, Planck JL, Kraus WL and Pascal JM The Zn3 domain of human poly(ADP-ribose) polymerase-1 (PARP-1) functions in both DNA-dependent poly(ADP-ribose) synthesis activity and chromatin compaction. J Biol Chem 285 : 18877-18887, 2010.

10. Liu JF and Silver DP: Poly ADP-ribose polymerase inhibitors: science and current clinical development. Curr Opin Oncol 22 567-572, 2010.

11. Lord CJ and Ashworth A: The DNA damage response and cancer therapy. Nature 481: 287-294, 2012

12. Javle M and Curtin NJ: The role of PARP in DNA repair and its therapeutic exploitation. Br J Cancer 105: 1114-1122, 2011.

13. Horton JK and Wilson SH: Hypersensitivity phenotypes associated with genetic and synthetic inhibitor-induced base excision repair deficiency. DNA Repair 6: 530-543, 2007.

14. Ame JC, Spenlehauer C and de Murcia G: The PARP superfamily. Bioessays 26: 882-893, 2004.

15. Plummer ER and Calvert H: Targeting poly(ADP-ribose) polymerase: a two-armed strategy for cancer therapy. Clin Cancer Res 13: 6252-6256, 2007

16. Woodhouse BC and Dianov GL: Poly ADP-ribose polymerase-1: an international molecule of mystery. DNA Repair 7: 1077-1086, 2008.

17. Dębska S, Kubicka J, Czyżkowski R, Habib M and Potemski P PARP inhibitors - theoretical basis and clinical application. Postepy Hig Med Dosw 66: 311-321, 2012 (In Polish).

18. Sobin LH and Wittekind C: TNM classification of malignant tumours. 5th edition, Wiley-Liss Inc, New York, 2002.

19. Elston CW and Ellis IO: Pathological prognostic factors in breast cancer. I. The value of histological grade in breast cancer: experience from a large study with long-term follow-up. Histopathology 19: 403-410, 1991

20. Halon A, Materna V, Drag-Zalesinka M, Nowak-Markwitz E, Gansukh T, Donizy P, Spaczynski M, Zabel M, Dietel M, Lage H and Surowiak P: Estrogen receptor alpha expression in ovarian cancer predicts longer overall survival. Pathol Oncol Res 17: 511-518, 2011.
21. Halon A, Nowak-Markwitz E, Maciejczyk A, Pudelko M, Gansukh T, Györffy B, Donizy P, Murawa D, Matkowski R, Spaczynski M, Lage H and Surowiak P: Loss of estrogen receptor beta expression correlates with shorter overall survival and lack of clinical response to chemotherapy in ovarian cancer patients. Anticancer Res 31: 711-718, 2011.

22. Szczuraszek K, Halon A, Materna V, Mazur G, Wrobel T, Kuliczkowski K, et al: Elevated YB-1 expression is a new unfavorable prognostic factor in non-Hodgkin's lymphomas. Anticancer Res 31: 2963-2970, 2011.

23. Remmele W and Stegner HE: Recommendation for uniform definition of an immunoreactive score (IRS) for immunohistochemical estrogen receptor detection (ER-ICA) in breast cancer tissue. Pathologe 8: 138-140, 1987 (In German).

24. Lage H and Dietel M: Multiple mechanisms confer different drugresistant phenotypes in pancreatic carcinoma cells. J Cancer Res Clin Oncol 128: 349-357, 2002.

25. Osheroff N, Corbert AH and Robinson MJ: Mechanism of action of topoisomerase II-targeted antineoplastic drugs. Adv Pharmacol 29B: 105-126, 1994.

26. Wichert A, Stege A, Midorikawa Y, Holm PS and Lage H: Glypican-3 is involved in cellular protection against mitoxantrone in gastric carcinoma cells. Oncogene 23: 945-955, 2004.

27. Kellner U, Hutchinson L, Seidel A, Lage H, Danks MK, Dietel $\mathrm{M}$ and Kaufmann SH: Decreased drug accumulation in a mitoxantrone-resistant gastric carcinoma cell line in the absence of P-glycoprotein. Int J Cancer 71: 817-824, 1997.

28. Lage H, Perlitz C, Abele R, Tampe R, Dietel M, Schadendorf D and Sinha P: Enhanced expression of human ABC-transporter tap is associated with cellular resistance to mitoxantrone. FEBS Lett 503: 179-184, 2001.

29. Lage H: Molecular analysis of therapy resistance in gastric cancer. Dig Dis 21: 326-338, 2003.

30. Györffy B, Surowiak P, Kiesslich O, Denkert C, Schäfer R, Dietel M and Lage H: Resistance prediction profile for eleven anticancer agents at clinical concentrations based on the gene expression pattern of thirty cell lines. Int J Cancer 118: 1699-1712, 2006.

31. Altan N, Chen Y, Schindler M and Simon SM: Defective acidification in human breast tumor cells and implications for chemotherapy. J Exp Med 187: 1583-1598, 1998.

32. Vickers PJ, Dickson RB, Shoemaker R and Cowan KH: A multidrug-resistant MCF-7 human breast cancer cell line which exhibits cross-resistance to antiestrogens and hormone-independent tumor growth in vivo. Mol Endocrinol 2: 886-892, 1988.

33. Ke W, Yu P, Wang J, Wang R, Guo C, Zhou L, Li C and Li K: MCF-7/ADR cells (re-designated NCI/ADR-RES) are not derived from MCF-7 breast cancer cells: a loss for breast cancer multidrug-resistant research. Med Oncol (Suppl) 1: S135-S141, 2011.

34. Domagala P, Huzarski T, Lubinski J, Gugala K and Domagala W: PARP-1 expression in breast cancer including BRCA1-associated, triple negative and basal-like tumors: possible implications for PARP-1 inhibitor therapy. Breast Cancer Res Treat 127: 861-869, 2011.

35. von Minckwitz G, Müller BM, Loibl S, Budczies J, Hanusch C, Darb-Esfahani S, et al: Cytoplasmic poly(adenosine diphosphate-ribose) polymerase expression is predictive and prognostic in patients with breast cancer treated with neoadjuvant chemotherapy. J Clin Oncol 29: 2150-2157, 2011.

36. Rojo F, Garcia-Parra J, Zazo S, Tusquets I, Ferrer-Lozano J, Menendez S, et al: Nuclear PARP-1 protein overexpression is associated with poor overall survival in early breast cancer. Ann Oncol 23: 1156-1164, 2012

37. Ozretic L, Rhiem K, Huss S, Wappenschmidt B, Markiefka B, Sinn P, Schmutzler RK and Buettner R: High nuclear poly(adenosine diphosphate-ribose) polymerase expression is predictive for BRCA1- and BRCA2-deficient breast cancer. J Clin Oncol 29: 4586-4588, 2011.

38. Surowiak P, Materna V, Matkowski R, Szczuraszek K, Kornafel J, Wojnar A, Pudelko M, et al: Relationship between the expression of cyclooxygenase 2 and MDR1/P-glycoprotein in invasive breast cancers and their prognostic significance. Breast Cancer Res 7: R862-R870, 2005.

39. Surowiak P, Materna V, Györffy B, Matkowski R, Wojnar A, Maciejczyk A, et al: Multivariate analysis of oestrogen receptor alpha, pS2, metallothionein and CD24 expression in invasive breast cancers. Br J Cancer 95: 339-346, 2006.

40. Maciejczyk A, Szelachowska J, Ekiert M, Matkowski R, Hałoń A, Lage H and Surowiak P: Elevated nuclear YB1 expression is associated with poor survival of patients with early breast cancer. Anticancer Res 32: 3177-3184, 2012. 